\title{
Analysis of random nonlinear water waves: the Stokes-Woodward technique
}

\section{Tanos Elfouhaily ${ }^{a}$, Maminirina Joelson ${ }^{a}$, Stéphan Guignard ${ }^{a}$, Hubert Branger ${ }^{a}$, Donald R. Thompson ${ }^{b}$, Bertrand Chapron ${ }^{c^{*}}$ and Douglas Vandemark ${ }^{\mathrm{d}}$}

\author{
${ }^{a}$ Centre national de la recherche scientifique (CNRS), institut de recherche sur les phénomènes hors equilibre \\ (IRPHE), Marseille, France \\ ${ }^{\mathrm{b}}$ The Johns Hopkins University, Applied Physics Laboratory, 11100 Johns Hopkins Road, Laurel, MD 20723- \\ 6099, USA \\ cDépartement d'océanographie spatiale, IFREMER, centre de Brest, BP 70, 29280, Plouzané, France \\ dNASA Goddard Space Flight Center, Laboratory for Hydrospheric Processes, Wallops Island, VA 23337, USA \\ *: Corresponding author : Bertrand.Chapron@ifremer.fr
}

\begin{abstract}
A generalization of the Woodward's theorem is applied to the case of random signals jointly modulated in amplitude and frequency. This yields the signal spectrum and a rather robust estimate of the bispectrum. Furthermore, higher order statistics that quantify the amount of energy in the signal due to nonlinearities, e.g., wave-wave interaction in the case of water waves, can be inferred. Considering laboratory wind generated water waves, comparisons between the presented generalization and more standard techniques allow to extract the spectral energy due to nonlinear wave-wave interactions. It is shown that our analysis extends the domain of standard spectral estimation techniques from narrow-band to broad-band processes.
\end{abstract}

Keywords: Fluid mechanics; Mode coupling; Wave-wave interaction; Horizontal asymmetry; Vertical asymmetry; Bispectrum; Amplitude modulation; Frequency modulation 


\section{Introduction and issues}

Common observations indicate that waves propagating on the ocean surface exhibit a stochastic behavior. This is especially true for wind waves generated under turbulent air flows. The randomness is exhibited in terms of highly irregular behavior of such parameters as the amplitude and the frequency of the field. These observation lead to a description of water waves as a superposition of random waves. A recent study Joelson and Ramamonjiarisoa [2001] has demonstrated that wind waves cannot be characterized by a deterministic system dynamically affected by nonlinearities.

Elfouhaily et al. [1999] demonstrated that an inconsistency, commonly encountered in the literature, occurs when weakly nonlinear theories are used to simulate nonlinear water waves. Wave-wave interactions tend to increase the energy of short waves [Weber and Barrick, 1977; Barrick and Weber, 1977]. Thus, the final spectrum obtained by the nonlinear simulation is different from the input spectrum. The common mistake is to use an empirically determined spectrum as input to the nonlinear simulation despite the fact that the output spectrum will deviate considerably from the measurements. Examples of such misuse have already been identified in Elfouhaily et al. [1999, 2000] which are concerned with understanding the electromagnetic bias observed by radar altimeters over the ocean surface. The contribution by Elfouhaily et al. [1999] suggests the need for an input spectrum, termed the "bare" spectrum, devoid from any nonlinear interaction. The output spectrum is then obtained from nonlinear interactions of all the modes present at the input to form the so-called "dressed" spectrum. Unfortunately, the "bare" spectrum does not yield itself easily to measurement since nonlinear wave-wave interactions cannot be turned off during the measurement of the surface wave spectrum. Higher order hydrodynamic interactions Elfouhaily et al. [2001b] will distort the input

spectrum even further hampering the study of its effect on, among other things, several remote sensing parameters [see Elfouhaily et al., 2001a].

Our present study is concerned with a new formulation of surface wave modulations 
that accounts for the nonlinear random nature of the process. A new technique is developed in order to quantify the amount of energy present in the spectrum caused by the nonlinear wave-wave interaction and mode coupling. The starting point will be a convenient theorem stated by Woodward [1952] which approximates the spectrum of frequency modulated signals by the probability density function of the instantaneous frequencies. A recent application of this theorem was successfully implemented in the study of delay and Doppler effects in bistatically reflected electromagnetic signals from the ocean surface Elfouhaily et al. [2002]. The Doppler application is very straightforward application of Woodward's theorem, while understanding the characteristics of the delay, some extrapolation was required as discussed in Elfouhaily et al. [2002]. We therefore believe that new generalizations of Woodward's theorem could have a large impact on several fields concerned with random nonlinear processes; an example of which is the remote sensing application discussed above.

In the following section, we summarize the original Woodward's theorem [Woodward, 1952] which we shall generalize to include joint amplitude and frequency modulations. A development up to second order captures the bispectrum in addition to the usual power spectrum. We will then compare our development with experimental data obtained from a wind-wave tank, where a clear difference between the bare and the dressed spectra is shown. The bare spectrum is obtained under no wave-wave interactions which can also be understood as the occurrence of a family of random fundamental frequencies. The dressed spectrum, however, depicts the observable energy when wave-wave interactions are present, and therefore can be interpreted as the augmentation due to a family of random harmonics. 


\section{Mathematical formulation of randomly modulated signals}

In the general context of stochastic stationary functions, the theorem of Bochner [1960] reads

$$
\eta(t, \alpha)=\int_{\omega} \mathrm{d} N(\omega, \alpha) \exp (-\mathrm{i} \omega t)
$$

where $d N(\omega, \alpha)$ is a non-correlated random measure and $\alpha$ is the event variable. The random process $\eta(t, \alpha)$ is called narrow-banded or quasi-monochromatic if and only if the random measure $d N(\omega, \alpha)$ occupies a narrow spectral bandwidth. The domain of integration in (1) is then limited between lower and upper bounds surrounding the spectral content of the random measure. This narrow-banded process exhibits weakly random modulations and can be written under near-Gaussian statistics according to Cramer and Leadbetter [1967] as

$$
\eta(t, \alpha)=R(\alpha) \cos \left[\omega_{c} t+\psi(\alpha)\right]
$$

where $R(\alpha)$ and $\psi(\alpha)$ are time independent random variables with Rayleigh and uniform distributions, respectively. The pulsation $\omega_{c}=2 \pi f_{c}$ is a constant carrier frequency. The event parameter $\alpha$ as introduced in (1) will be omitted throughout the paper to simplify the equations. The weak random modulation is a typical characteristic of surface water waves [Kinsman, 1965]. According to Longuet-Higgins [1983]; Tayfun [1986], these oscillations can be well represented to first approximation by (2). However, this narrow-band formulation is insufficient to explain the complexity of water waves when higher-order statistics must be included due to the asymmetric behavior caused by nonlinear wave-wave interactions. In this case, large deviations from a narrow-band approximation can be observed especially under conditions of wind generated waves, and the random variables in (1) are no longer time independent [Middleton, 1996]. Under these conditions, the processes become broad band in nature.

In this study, we model random nonlinear surface waves as broad-band processes with the objective of properly characterizing the spectral content of these signals. 
A distinction will be made between spectral density due to nonlinearity as opposed to that when no wave-wave interactions are present. To achieve these goals, we will generalize Woodward's theorem [Woodward, 1952] to include amplitude modulations under moderately large indices of modulations.

\section{Woodward's theorem}

\subsection{Definition}

A frequency or phase modulated signal can be written as

$$
\eta(t)=A \cos \left[\omega_{c} t+\nu \phi\left(\frac{t}{\nu}\right)\right]
$$

where $\nu$, in this notation, represents the index of modulation and $A$ a constant amplitude. The pulsation $\omega_{c}$ is the carrier frequency with $\omega_{c}=2 \pi f_{c}$. Woodward's theorem states that the spectrum of a signal randomly modulated in frequency is proportional to the probability density function $\left(P_{\dot{\phi}}\right)$ of the modulating instantaneous frequency $\left(\frac{\mathrm{d} \phi}{\mathrm{d} t}=\dot{\phi}=2 \pi D(t)\right)$. The corresponding spectrum is then

$$
S(f) \approx \frac{A^{2}}{2} P_{\dot{\phi}}\left(f-f_{c}\right)
$$

where for simplicity negative frequencies have been folded onto positive frequencies since the signal under study is real.

\subsection{Proof}

Following Blachman and McAlpine [1969], the proof of Woodward's theorem may be sketched as follows: One can write the autocorrelation function of $\eta(t)$ in $(3)$ as

$$
\begin{aligned}
R(\tau) & =\left\langle\eta\left(t-\frac{\tau}{2}\right) \eta\left(t+\frac{\tau}{2}\right)\right\rangle \\
& =\frac{A^{2}}{2} \operatorname{Re}\left\langle\exp \left(\mathrm{i} \omega_{c} \tau+\mathrm{i} 2 \pi \int_{t-\frac{\tau}{2}}^{t+\frac{\tau}{2}} D\left(\frac{t}{\nu}\right) \mathrm{d} t\right)\right\rangle
\end{aligned}
$$


where $D(t)=\dot{\phi} / 2 \pi$ is the modulating instantaneous frequency of the random signal. If the index of modulation is very high $(\nu>>1)$, then one can demonstrate that [Blachman and McAlpine, 1969]

$$
\left\langle\exp \left(2 i \pi \int_{t-\frac{\tau}{2}}^{t+\frac{\tau}{2}} D\left(\frac{t}{\nu}\right) \mathrm{d} t\right)\right\rangle=\int_{-\infty}^{+\infty} P_{\dot{\phi}}(D) \exp (2 i \pi D \tau) \mathrm{d} D .
$$

The autocorrelation function becomes

$$
R(\tau)=\frac{A^{2}}{4}\left\{\int_{-\infty}^{+\infty} P_{\dot{\phi}}(D)\left\{\exp \left[2 i \pi\left(D+f_{c}\right) \tau\right]+\exp \left[-2 i \pi\left(D+f_{c}\right) \tau\right]\right\} \mathrm{d} D\right\}
$$

Woodward's theorem is then demonstrated by taking the Fourier transform of the autocorrelation function in (7) to yield (4) after spectral folding of the negative frequencies onto the positive ones.

\section{Generalization to joint amplitude and frequency modulations}

We now want to find an equivalence to Woodward's theorem in the frequency modulation context as stated by (4) but with an additional random modulation coming through the amplitude. In this context, the form of the signal to be studied is then

$$
\eta(t)=a\left(\frac{t}{\mu}\right) \cos \left(\omega_{c} t+2 \pi \int_{0}^{t} D\left(\frac{\tau}{\nu}\right) \mathrm{d} \tau\right)
$$

where $a\left(\frac{t}{\mu}\right)$ is a random process with an index of modulation $\mu$ and $D\left(\frac{\tau}{\nu}\right)$ is the

modulating random instantaneous frequency with index $\nu$. The autocorrelation function of this process is

$$
R(\tau)=\left\langle a\left(\frac{t-\frac{\tau}{2}}{\mu}\right) a\left(\frac{t+\frac{\tau}{2}}{\mu}\right) \exp \left(\mathrm{i} \omega_{c} \tau+2 i \pi \int_{t-\frac{\tau}{2}}^{t+\frac{\tau}{2}} D\left(\frac{t}{\nu}\right) \mathrm{d} t\right)\right\rangle .
$$

If the modulation indices $\mu$ and $\nu$ are large enough [Crandall, 1963], a power series expansion of (9) can be performed up to second order to yield

$$
R(\tau) \approx\left\langle\left(a-\frac{\dot{a} \tau}{2 \mu}\right)\left(a+\frac{\dot{a} \tau}{2 \mu}\right) \exp \left(\mathrm{i} \omega_{c} \tau+2 \mathrm{i} \pi \tau\left[D(t / \nu)+\frac{2 t \dot{D}(t / \nu)}{\nu}\right]\right)\right\rangle .
$$


The spectrum corresponding to (10) is then

$$
S(f) \approx \frac{1}{2} \int\left[a^{2}-\frac{\dot{a}^{2}}{4 \mu^{2}}\left(\frac{\partial^{2}}{\partial \omega^{2}}\right)\right] P\left(a, \dot{a}, \omega-\frac{\dot{\omega} t}{\nu}, \dot{\omega}\right) \mathrm{d} a \mathrm{~d} \dot{a} \mathrm{~d} \dot{\omega},
$$

where $\omega=2 \pi f$. The generalization of Woodward's theorem requires knowledge of the joint distribution of four random processes as opposed to that of one process in the original theorem. This generalization to a four dimensional distribution in (11) is generally not useful since it is impractical to estimate such multidimensional distributions from the time series of the signal itself. We shall now present a more practical formulation of this generalization where a joint distribution with fewer dimensions is required.

\section{The Stokes-Woodward technique}

\subsection{Statistical modulation}

As mentioned above, a brute force generalization of Woodward's theorem as in (11) is inefficient and may even be unstable if the time series under study is not long or stationary enough. It is easy to notice that (11) reaches a very practical limit if both indices of amplitude and frequency modulations, $\mu$ and $\nu$ respectively, are nearly infinite. In this limit, (11) reduces to what we call the bare spectrum

$$
S_{\text {bare }}(f) \approx \frac{1}{2} \int a^{2} P(a, f) \mathrm{d} a
$$

This approximation is very practical and requires only the estimation of a two-

dimensional histogram. However, this practicality is gained at the expense of neglecting the temporal modulations of the amplitude and frequency within the scale of the dominant time period. In other words, the temporal modulations is slow and interpreted as random from one period to the next. More simply stated, the high index approximation in (12) is actually an exact formulation for a random process of this form

$$
\eta(t)=a \cos (\omega t+\theta)
$$


where $a, \omega$, and $\theta$ are three time-independent random variables. The amplitude $a$ and the pulsation $\omega=2 \pi f$ can be statistically dependent, while $\theta$ is a uniformly distributed phase and independent of these other variables. This requirement on the uniformity of the phase guarantees that the signal is stationary and therefore that the autocorrelation and the spectral functions are univariate. The "bare" subscript in (12) refers to the fact that the process in (13) has lost all nonlinearity or phase coupling of harmonics.

\subsection{Temporal and statistical modulation}

The high index limit in (12) is very illustrative and permits the reformulation of our newly generalized theorem into a simpler form. The time modulation present in (12) can now be replaced by a random modulation as in (13). Let us reformulate the modulation as

$$
\eta(t)=[a+\Delta a(t)] \cos [\omega t+\Delta \phi(t)+\theta]
$$

where the time dependence is explicitly shown in addition to the implicit random dependence of all the parameters except the time variable. The key representation of our simplified expression is in the time dependence of the modulation that can be expanded in Fourier series about the random frequency $\omega$ as

$$
\begin{aligned}
\Delta a(t) & =\alpha_{c} \cos (\omega t+\theta)+\alpha_{s} \sin (\omega t+\theta)+\ldots \\
\Delta \phi(t) & =\beta_{c} \cos (\omega t+\theta)+\beta_{s} \sin (\omega t+\theta)+\ldots
\end{aligned}
$$

The signal in (14) can be further expanded keeping only terms of linear order in the parameters $\alpha_{c}, \alpha_{s}, \beta_{c}$, and $\beta_{s}$ to find a more poignant form given by

$$
\eta(t)=\lambda+a \cos [\omega t+\theta]+\alpha \cos [2(\omega t+\theta)]+\beta \sin [2(\omega t+\theta)]+\ldots
$$


where the coefficients $\alpha$ and $\beta$ of the second harmonic terms in (16) are related to the coefficients in (15) by

$$
\begin{aligned}
\lambda & =\frac{a \beta_{s}+\alpha_{s}}{2} \\
\alpha & =\frac{a \beta_{s}+\alpha_{c}}{2} \\
\beta & =\frac{\alpha_{s}-a \beta_{c}}{2} .
\end{aligned}
$$

We remind the reader that all parameters in (16) are random variables except the time variable. Finally, not included in our analysis is the constant term $\lambda$. We save discussions of the significance of this term for a future publication. There is no influence of the presence or the absence of the constant term in the dataset selected for illustration in the current paper.

Equation (16) represents a generalization to the Stokes wave in two regards. First, all parameters are random as opposed to the Stokes wave where all parameters must stay constant. Second, the $\sin ()$ term generates asymmetries in the profile that are not included in a standard Stokes waveform. Its resemblance with the Stokes wave, however, did influence our choice for naming our analysis method, which uses Woodward's theorem and Stokes-like waveforms, the "Stokes-Woodward" technique. The random variables $\alpha$ and $\beta$ explain the asymmetries of the waveform with respect to a horizontal and vertical axes, respectively. These asymmetries appear in a random manner on the scale of the period of the wave as depicted by the random amplitude $a$ and random frequency $\omega$.

Similar to equation (13), the random Stokes-like waveforms in (16) yields a spectrum of the Woodward type but in a more accessible form than in (11). This form is given by

$$
S_{\text {dressed }}(f) \approx S_{\text {bare }}(f)+\frac{1}{2} \int \alpha^{2} P(\alpha, f / 2) \mathrm{d} \alpha+\frac{1}{2} \int \beta^{2} P(\beta, f / 2) \mathrm{d} \beta .
$$

In this final form, two single integrals are needed in addition to the single integration 
over the amplitude already present in the "bare" spectrum of (12). We associate the spectrum in (18) with the "dressed" spectrum discussed in the introduction, and interpret the terms added to the bare spectrum as contributing to the energy increase at higher frequencies due to the nonlinearities or mode coupling. This energy augmentation therefore provides the difference between the bare and the dressed spectra as discussed in [Elfouhaily et al., 1999]. The difference can also be assimilated with the bicoherence function of phase coupling as introduced by Kim and Powers [1979] and utilized by Ochi and Ahn [1994]. Indeed, energy of phase coupling to second order is another manifestation of the bispectrum defined as the Fourier transform of the skewness function using a third order cumulant. The bispectrum is defined as the 2D Fourier transform of the bivariate skewness function

$$
R\left(\tau_{1}, \tau_{2}\right)=\left\langle\eta(t) \eta\left(t+\tau_{1}\right) \eta\left(t+\tau_{2}\right)\right\rangle
$$

The description of the vertical and horizontal asymmetries of the waveform opens the way for the definition of a very robust bispectral estimate derived from our Stokes-Woodward technique. The bispectrum takes the form

$$
B(f)=B(f, f / 2) \approx \frac{1}{4} \iint \alpha a^{2} P(\alpha, a, f / 2) \mathrm{d} \alpha \mathrm{d} a+\mathrm{i} \frac{1}{4} \iint \beta a^{2} P(\beta, a, f / 2) \mathrm{d} \beta \mathrm{d} a
$$

where now two three-dimensional histograms are needed in order to evaluate the double integration over the amplitude and the asymmetries. It is no surprise that the height (or vertical) asymmetry $\alpha$ yield the real part of the bispectrum while the period (or horizontal) asymmetry $\beta$ yield the imaginary part. We shall show in the next section that the estimation of the "bare" and "dressed" spectra as well as the bispectrum is very robust and can lead to interesting interpretations of the surface wave physics. 


\section{Comparison with data}

\subsection{Experimental data}

The Stokes-Woodward technique is now tested using a time series generated by measuring the height of the water surface at a fixed point in a wind-wave tank. The signals were obtained from the Ocean-Atmosphere Interaction facility in Marseille with a capacitance wave gauge. The pool dimensions in the tank are 40x3x1 meters for the length, width, and depth, respectively. The air tunnel's ceiling is $1.5 \mathrm{~m}$ above the water surface. For a detailed description of the flume-tank, the reader is referred to Coantic and Favre [1974]. In order to generate highly nonlinear waves where mode coupling should be significant, we chose to sample wind-generated waves under high wind conditions with wind speeds higher than $10 \mathrm{~m} / \mathrm{s}$ with a fetch of $25 \mathrm{~m}$ but without paddle waves. Long time series were acquired at high frequency sampling. Duration and sampling rate were on the order of 30 minutes and $100 \mathrm{~Hz}$, respectively. Figure 1 shows a short sample of the time series of surface elevations. High modulations and group occurrences are easily visible in this short segment. In order to estimate the instantaneous amplitude $a$, frequency $f$, height asymmetry $\alpha$ and period asymmetry $\beta$ in (16), we implemented the zero-crossing algorithm as explained in Molinaro and Sergeyev [2001]. Ambiguities related to the definition of the instantaneous frequency [see Oliveira and Barroso, 2000] are not dealt with here, but will be left for later contributions when more exact algorithms will be used. We mention that the zero-crossing algorithm is well defined as long as the broad-band signal has a unimodal spectrum with no low frequency components or high noise contaminations. Practical estimators for the four 
random variables, $a, \omega=2 \pi / T$, $\alpha$, and $\beta$ are taken as follows:

$$
\begin{aligned}
a & =\frac{M-m}{2} \\
T & =T_{1}+T_{2} \\
\alpha & =\frac{M+m}{2} \\
\beta & =a \frac{\pi}{2} \frac{T_{1}-T_{2}}{T_{1}+T_{2}},
\end{aligned}
$$

where $M$ and $m$ are the maximum and the minimum of the signal over a period $T$ defined as the sum of two consecutive semi-periods $T_{1}$ and $T_{2}$. The semi-periods are the durations between two consecutive zero-crossings. Figure 2 shows an illustration, on the scale of a period, the definition of the instantaneous parameters used in (21).

Figure 2.

\subsection{Analysis results}

Figure 3 illustrates the stability of the estimators for the asymmetry parameters as shown in terms of their normalized histograms. The height asymmetry $\alpha$ is normalized by the amplitude $a$ where the period asymmetry $\beta$ is normalized by $a \frac{\pi}{2}$. A striking difference is easily noticeable between these histograms and the Gaussian distributions shown for reference by the solid lines in the figure. Indeed, the histograms seem to be highly peaked with some skewness. The kurtosis is very high and on the order of 4 and 6 for the height $(\alpha)$ and period $(\beta)$ asymmetries, respectively. The ragged line in Figure 4 shows the Fourier spectrum of the time series. Using a standard spectrum such as this, one is not able to differentiate between energies coming from linear or nonlinear waves. The Woodward spectrum as defined in (4), is the highest solid curve in Figure 4. One can see that this curve over-estimates the measured spectrum because of the underlying Woodward assumption that, on the contrary to the real signal, the amplitude is not modulated, and that only frequency modulation is present. The over-estimation of the spectral tail is therefore due to the fact that the amplitude is modulated and also correlated with the frequency in such a manner that high frequency waves have less 
energy. The lowest solid line in Figure 4 is the "bare" spectrum as defined by (12) where both amplitude and frequency modulations are accounted for with no asymmetries or phase coupling. It is instructive to notice that the "bare" spectrum underestimates the energy in the tail of the measured spectrum. This under estimation is due to the implicit assumption in (12) that the indices of modulations are very high and therefore any nonlinearity caused by time-dependent modulations is neglected. The "dressed" spectrum (18) is shown in figure 4 by the dashed-dotted curve. This curve accounts for most of the energy present in the measured Fourier spectrum. Both vertical and horizontal asymmetries contribute to the energy at high frequencies. This can be seen by the fact that the dashed curve shown in figure 4, which includes modulation of only the height $(\alpha)$ asymmetry, under estimates the high-energy content of the measured spectrum. It therefore seems apparent that our Stokes-Woodward technique explains the energy due to mode coupling. A direct measure of the coupling can also be achieved by calculating the bispectrum as defined in (20). The result of this computation is shown in Figure 5 in terms of its modulus square. The high stability of the bispectral estimate up to a quite high frequency demonstrates the wide domain of applicability of our technique. The peak present in the bispectrum indicates that many frequencies or harmonics are coupled with their fundamental counterparts at lower frequencies. It is therefore important to treat the ensemble of frequencies as two correlated random sets. Based on the results of the comparisons presented in this section, we believe our technique can accurately interpret the occurrences of instantaneous amplitudes, frequencies, vertical and horizontal asymmetries. This demonstrates the capability to detecting mode coupling in spectral domain of second statistical order without performing bispectral analysis.

Figure 5. 


\section{Conclusion}

A generalization of Woodward's theorem is successfully obtained by including random amplitude modulations in addition to frequency modulations. The original theorem stated that a good approximation of the energy spectrum of a frequency modulated signal is the probability density function of the instantaneous frequencies when the index of modulation is high. Our generalization starts by including the random amplitude modulation which yield a simple spectrum expressed as a single integral over the instantaneous amplitudes and the joint distribution of amplitude and frequency as shown in (12). It is noted that this spectrum is devoid of any nonlinearity or mode coupling because over the scale of a characteristic period, the wave is considered as simply harmonic (a sine wave). Asymmetries in the wave profile must be introduced in order to capture residual energy not explained by the "bare" spectrum. To account for this residual energy, we have proposed a second generalization of Woodward's theorem that utilizes a Stokes-like waveform in which all the parameters are random except the time variable. Our procedure is termed the Stokes-Woodward technique since it combines a generalization of Woodward's theorem with a Stokes-like random wave profile.

The second generalization provides a practical formulation for the "dressed" spectrum where nonlinearities up to the second order are included (18). This second order coupling between modes initiates the existence of the bispectrum which can be

formulated as in (20). It is demonstrated that when the Stokes-Woodward technique is applied to a time series of water-wave surface elevations, it discriminates between the "bare" and "dressed" spectrum, and also provides a robust estimate of the bispectrum. We recommend that the bare spectrum be used at the input of nonlinear system simulators as originally cautioned in Elfouhaily et al. [1999].

Applications of the Stokes-Woodward technique will have great benefit in the analysis of nonlinear random processes present in several science fields. For example, it 
can readily be applied to remote sensing signals as already demonstrated by [Elfouhaily et al., 2002] even with the original formulation of Woodward's theorem. 


\section{References}

Barrick, D., and B. Weber, On the nonlinear theory for gravity waves on the ocean's surface. part ii: Interpretation and applications, J. Phys. Oceanogr., 7, 11-21, 1977.

Blachman, N. M., and G. A. McAlpine, The spectrum of a high-index fm waveform: Woodward's theorem revisited, IEEE Trans. Comm. Tech., COM-17, 201-207, 1969.

Bochner, S., Harmonic analysis and the Theory of probability, Univ.California Press, California USA, 1960.

Coantic, M., and A. Favre, Activities in and preliminary results of air-sea interaction research at I.M.S.T, Adv.Geophys., 16, 391-405, 1974.

Cramer, H., and M. R. Leadbetter, Stationary and Related Stochastic Processes, John-Whiley, New York, USA, 1967.

Crandall, S. H., Perturbation techniques for random vibration of nonlinear systems, $J$. Acc. Soc. America, 35, 1700-1705, 1963.

Elfouhaily, T., D. R. Thompson, B. Chapron, and D. Vandemark, Weakly non-linear theory and sea state bias estimations, J. Geophys. Res., 104, 7641-7647, 1999.

Elfouhaily, T., D. R. Thompson, B. Chapron, and D. Vandemark, Improved electromagnetic bias theory, J. Geophys. Res., 105, 1299-1310, 2000.

Elfouhaily, T., D. R. Thompson, B. Chapron, and D. Vandemark, Improved electromagnetic bias theory: Inclusion of hydrodynamic modulations, J. Geophys. Res., 106, 4655-4664, 2001a.

Elfouhaily, T., D. R. Thompson, B. Chapron, and D. Vandemark, Higher-order hydrodynamic modulation: theory and applications for ocean waves, Proc. $R$. Soc. Lond. A., 457, 2585-2608, 2001b. 
Elfouhaily, T., D. Thompson, and L. Linstrom, Delay-Doppler analysis of bistatically reflected signals from the ocean surface: theory and application, IEEE Trans. Geosci. and Remote Sens., 40, 560-573, 2002.

Joelson, M., and A. Ramamonjiarisoa, A nonlinear second-order stochastic model of ocean surface waves, Oceanologica Acta, 24, 1-7, 2001.

Kim, Y. C., and E. J. Powers, Digital bispectral analysis and its applications to nonlinear wave interactions, IEEE Trans. Plasma Sci., PS-7, 120-131, 1979.

Kinsman, B., Wind Waves: their generation and propagation in Ocean surface, Prentice-Hall Inc., Englewood Cliffs, NJ, USA, 1965.

Longuet-Higgins, M. S., On the joint distribution of wave periods and amplitudes in random wave field, Proc. R. Soc. Lond. A., 389, 241-258, 1983.

Middleton, D., An introduction to statistical communication theory, 3 ed., IEEE Press, Piscataway, New Jersey, 1996, 1152 pages.

Molinaro, A., and Y. D. Sergeyev, An efficient algorithm for the zero crossing detection in digitized measurement signal, Measurement, 30, 187-196, 2001.

Ochi, M. K., and K. Ahn, Probability distribution applicable to non-gaussian random processes, Prob. Engng. Mech., 9, 255-264, 1994.

Oliveira, P. M., and V. Barroso, Definitions of instantaneous frequency under physical constraints, J. Franklin Inst., 337, 303-316, 2000.

Tayfun, M. A., On narrow-band representation of ocean waves, 1. Theory, J. Geophys. Res., 91, 7743-7752, 1986.

Weber, B., and D. Barrick, On the nonlinear theory for gravity waves on the ocean's surface. part i: Derivations, J. Phys. Oceanogr., 7, 3-10, 1977.

Woodward, P. M., The spectrum of random frequency modulation, Technical 
memorandum, Telecommunications Research Establishement, Great Malvern, Worcs., England, 1952, 666 pages.

T. Elfouhaily, M. Joelson, S. Guignard, H. Branger Centre National de la Recherche Scientifique, CNRS-IRPHE-IOA, Luminy Case 903, 13288 Marseille Cedex 9 - France. (e-mail: elfouhaily@pollux.irphe.univ-mrs.fr)

D.R. Thompson, The Johns Hopkins University, Applied Physics Laboratory, 11100 Johns Hopkins Road, Laurel, MD 20723-6099, USA. (e-mail: donald.thompson@jhuapl.edu)

B. Chapron, Département d'Océanographie Spatiale, IFREMER, centre de Brest, BP 70, 29280 Plouzané, France. (e-mail: Bertrand.Chapron@ifremer.fr)

D. Vandemark, NASA Goddard Space Flight Center, Laboratory for Hydrospheric Processes, Wallops Island, VA 23337, USA. ( e-mail: vandemark@gsfc.nasa.gov) Received

Submitted to Comptes Rendus de l'Académie de Science, August 2002.

This manuscript was prepared with AGU's LTEX macros v4, with the extension package 'AGU'+' by P. W. Daly, version 1.6b from 1999/08/19. 


\section{Figure Captions}

Figure 1. Time series of the surface elevation sampled at $100 \mathrm{~Hz}$. High modulations are clearly present.

Figure 2. Sketch plot to show the definitions of the paratemeters $\left(M, m, T_{1}, T_{2}\right)$ used to estimate the instantaneous amplitude $a=(M-m) / 2$, period $T=T_{1}+T_{2}$, vertical $\alpha=(M+m) / 2$ and horizontal $\beta=a \pi\left(T_{1}-T_{2}\right) /\left(T_{1}+T_{2}\right) / 2$ asymmmetries.

Figure 3. Normalized histograms of the normalized horizontal or height (dashed) and vertical or period (dashed-dotted) asymmetries. The normalization of the asymmetry parameters refers to rendering $\alpha$ and $\beta$ dimensionless by dividing by the amplitude. The solid curves are Gaussian distributions plotted for comparison.

Figure 4. The ragged line shows the Fourier spectrum of the experimental wave profile. The upper solid curve represents the Woodward spectrum as defined in (4). The lower solid line is the "bare" spectrum where wave-wave nonlinear interaction are neglected. The dashed curve is the augmentation of the "bare" spectrum by the height asymmetries alone. The dashed dotted curve is the total "dressed" spectrum where both horizontal and vertical asymmetries are included.

Figure 5. Modulus square of the Bispectrum which shows a significant peak indicating the mode coupling of the frequencies. These plots are normalized by their corresponding total integral. The dashed ragged curve is the bispectral estimated based on simple Fourier analysis. The solid curve is the bispectral estimate as obtained by our Stokeswoodward techinque. 
Figures

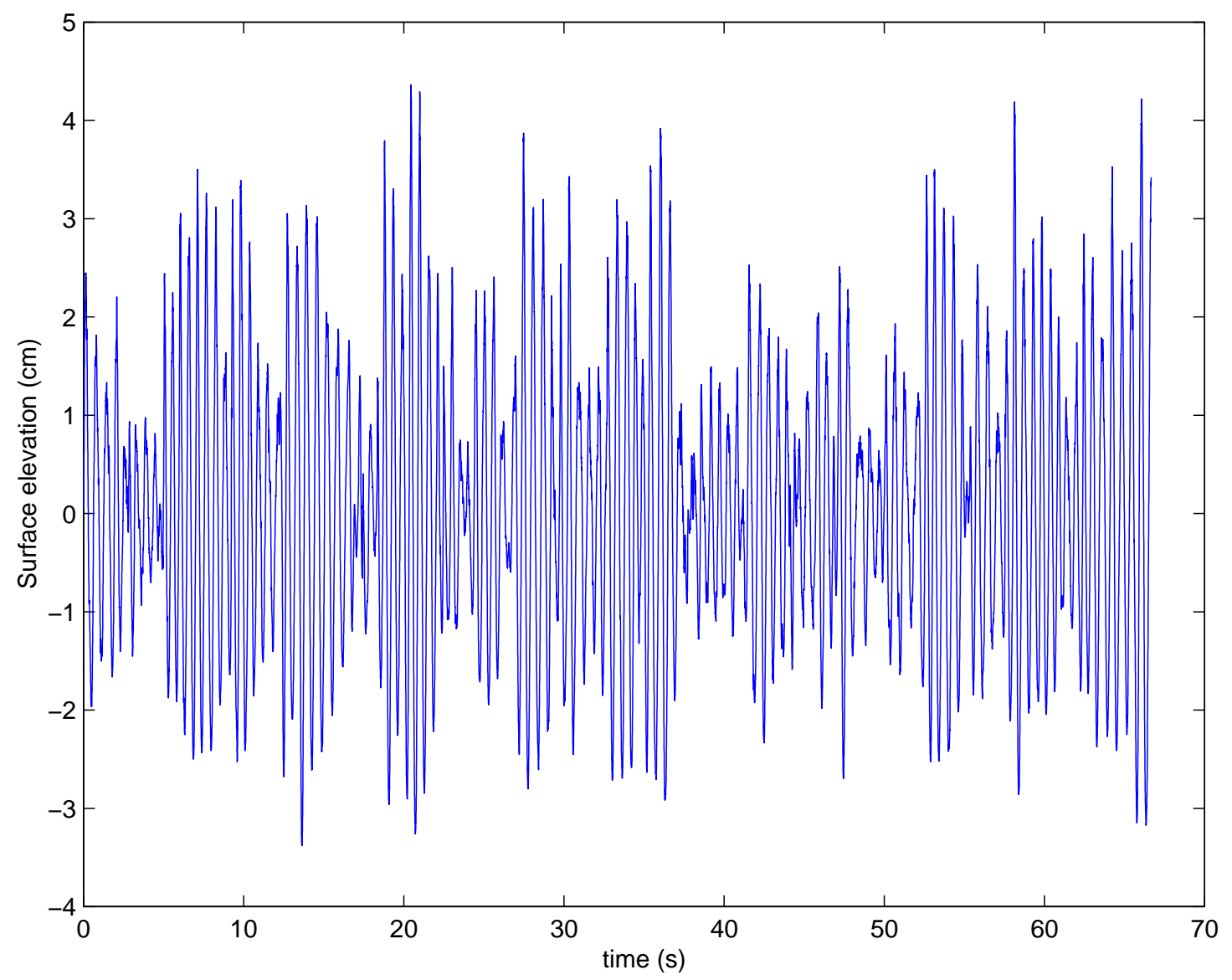

Figure 1. Time series of the surface elevation sampled at $100 \mathrm{~Hz}$. High modulations are clearly present. 


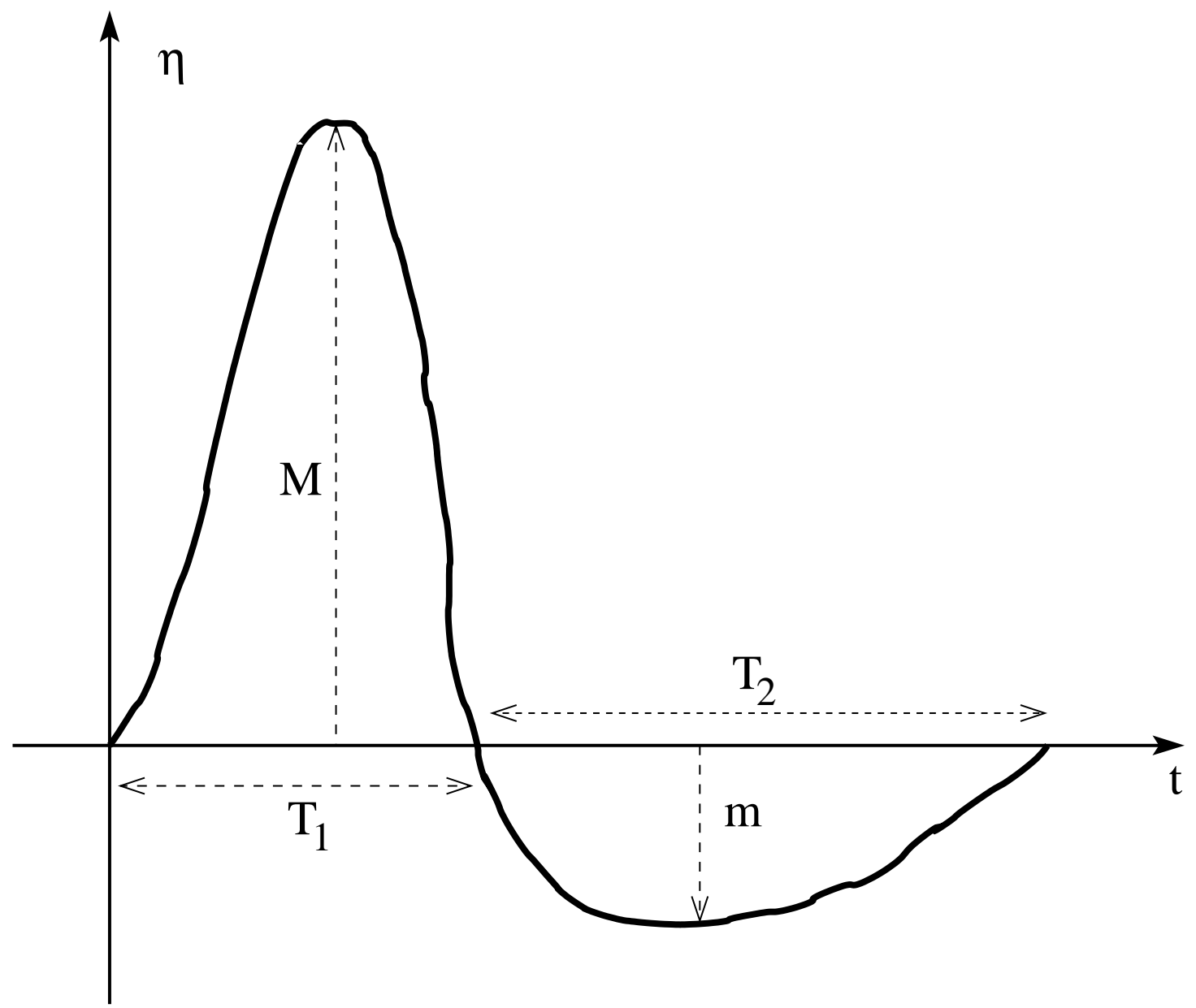

Figure 2. Sketch plot to show the definitions of the paratemeters $\left(M, m, T_{1}, T_{2}\right)$ used to estimate the instantaneous amplitude $a=(M-m) / 2$, period $T=T_{1}+T_{2}$, vertical $\alpha=(M+m) / 2$ and horizontal $\beta=a \pi\left(T_{1}-T_{2}\right) /\left(T_{1}+T_{2}\right) / 2$ asymmmetries. 


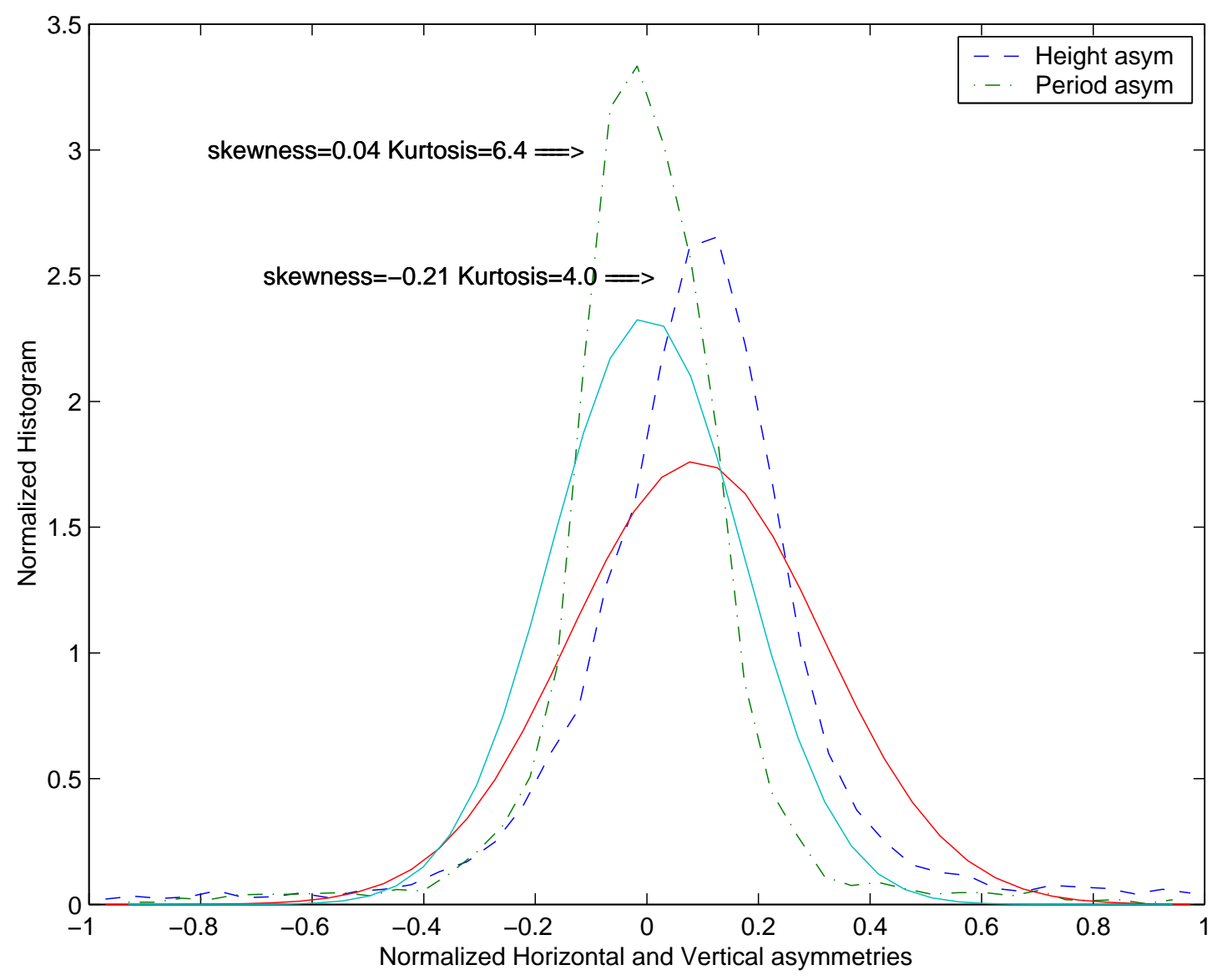

Figure 3. Normalized histograms of the normalized horizontal or height (dashed) and vertical or period (dashed-dotted) asymmetries. The normalization of the asymmetry parameters refers to rendering $\alpha$ and $\beta$ dimensionless by dividing by the amplitude. The solid curves are Gaussian distributions plotted for comparison. 


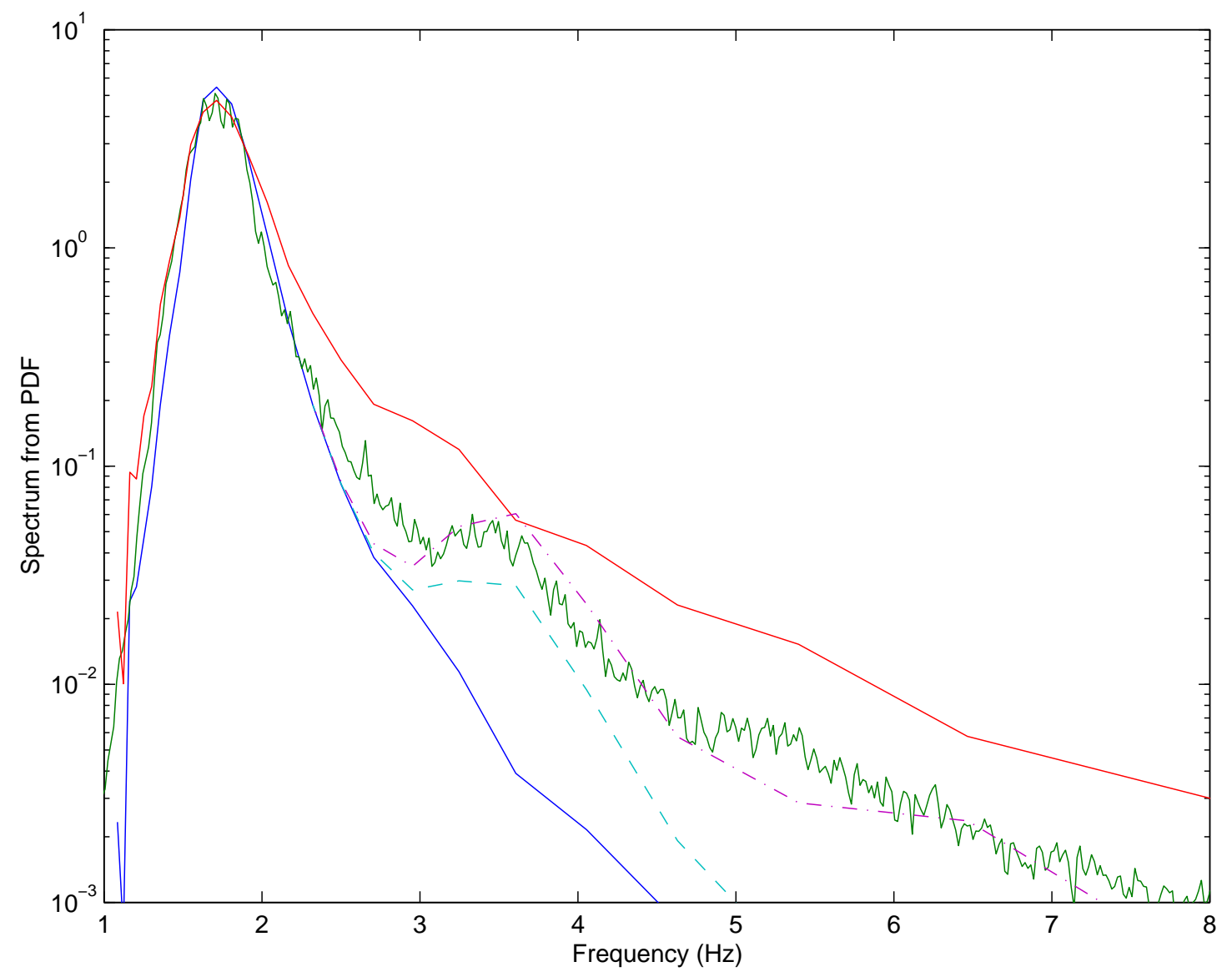

Figure 4. The ragged line shows the Fourier spectrum of the experimental wave profile. The upper solid curve represents the Woodward spectrum as defined in (4). The lower solid line is the "bare" spectrum where wave-wave nonlinear interaction are neglected. The dashed curve is the augmentation of the "bare" spectrum by the height asymmetries alone. The dashed dotted curve is the total "dressed" spectrum where both horizontal and vertical asymmetries are included. 


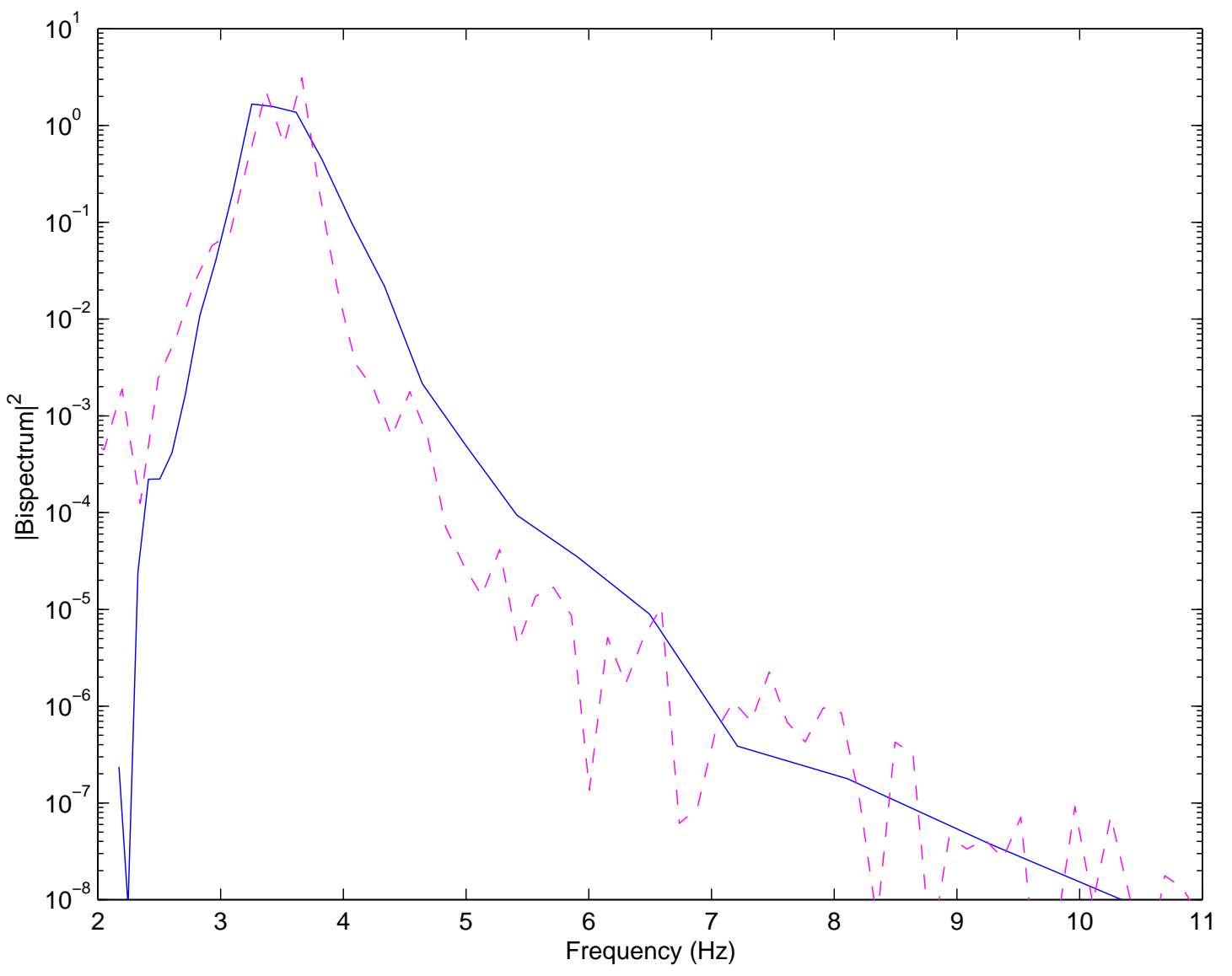

Figure 5. Modulus square of the Bispectrum which shows a significant peak indicating the mode coupling of the frequencies. These plots are normalized by their corresponding total integral. The dashed ragged curve is the bispectral estimated based on simple Fourier analysis. The solid curve is the bispectral estimate as obtained by our Stokes-woodward techinque. 